\title{
Effects of subliminal hints on insight problem solving
}

\author{
Masasi Hattori • Steven A. Sloman • Ryo Orita
}

Published online: 8 February 2013

(C) Psychonomic Society, Inc. 2013

\begin{abstract}
Two experiments tested a total of 509 participants on insight problems (the radiation problem and the nine-dot problem). Half of the participants were first exposed to a 1min movie that included a subliminal hint. The hint raised the solution rate of people who did not recognize it. In addition, the way they solved the problem was affected by the hint. In Experiment 3, a novel technique was introduced to address some methodological concerns raised by Experiments 1 and 2 . A total of 80 participants solved the 10-coin problem, and half of them were exposed to a subliminal hint. The hint facilitated solving the problem, and it shortened the solution time. Some implications of subliminal priming for research on and theorizing about insight problem solving are discussed.
\end{abstract}

Keywords Subliminal priming · Implicit cognition ·

Convergence problem $\cdot$ Nine-dot problem $\cdot$ Ten-coin problem

A striking aspect of insight problem solving occurs when people reach an impasse despite having all the knowledge required for a solution. To explain how people resolve impasses, some theorists emphasize changes in heuristic search of the problem space (e.g., Chronicle, MacGregor, \&

Electronic supplementary material The online version of this article (doi:10.3758/s13423-013-0389-0) contains supplementary material, which is available to authorized users.

M. Hattori $(\square)$

Department of Psychology, Ritsumeikan University,

56-1 Toji-in Kitamachi, Kita-ku,

Kyoto 603-8577, Japan

e-mail: hat@1t.ritsumei.ac.jp

S. A. Sloman

Department of Cognitive, Linguistic, and Psychological Sciences,

Brown University, Providence, RI, USA

R. Orita

Department of Psychology, Ritsumeikan University, Kyoto, Japan
Ormerod 2004; Kaplan \& Simon, 1990), while others emphasize changes in the distribution of activation in memory (e.g., Ohlsson, 2011; Seifert, Meyer, Davidson, Patalano, \& Yaniv, 1995). The former view puts the theoretical workload on controlled, attention-demanding, and conscious processes, the latter view on automatic, effortless, and unconscious processes such as the spread of activation.

Gick and Holyoak (1980) long ago demonstrated the importance of awareness in accessing relevant information. They used Duncker's (1945) radiation problem, which requires devising a method to destroy an inoperable tumor in a patient's stomach by a kind of ray without causing any serious damage to surrounding healthy tissue. Presenting a story that was structurally analogous to the solution (i.e., to converge multiple low-intensity rays) did not enhance participants' success unless it was explicitly presented as a hint. Lockhart, Lamon, and Gick (1988) claimed that conceptual processing of the prime triggers an awareness of the relevance of the information and is required for transfer. On the other hand, Maier (1931) observed that people can make use of a hint incidentally given to them to solve the two-string (pendulum) problem even if they were not aware of the hint. Schunn and Dunbar (1996) also showed that people can transfer their knowledge analogically to help solve even a complex problem without awareness that they are doing so.

We examine the contribution of hints that are primed subliminally to solving insight problems. Like Maier's (1931), most problem-solving studies have been concerned with transfer from analogical tasks, or supraliminal priming, in which an individual is fully aware of the stimulus, although he or she may not be aware that it is actually a hint. This method, however, may be susceptible to both memory failures (e.g., participants may merely have forgotten being aware) and demand characteristics (Bowden, 1997). Subliminal priming has neither disadvantage and, thus, offers an ideal method for examining the role of awareness in the resolution of impasses. To our knowledge, however, 
this technique has not been applied to insight problem solving, except by Nishimura and Suzuki (2006), who report, without describing their methods in detail, subliminal priming of the solution time for the T Puzzle. ${ }^{1}$

Evidence does suggest that subliminal priming affects people's preferences (Kunst-Wilson \& Zajonc, 1980), interpersonal judgments (Bargh \& Pietromonaco, 1982), brand choice (Karremans, Stroebe, \& Claus, 2006), motivation in classrooms (Radel, Sarrazin, Legrain, \& Gobancé, 2009), and even higher-order goals (Légal, Chappé, Coiffard, \& Villard-Forest, 2012). There is, however, not yet agreement on the definition of a perceptual threshold (Snodgrass, Bernat, \& Shevrin, 2004), and some researchers still dismiss subliminal perception (see Holender, 1986). Most studies define subliminal perception in terms of subjective report. In this study, we use a more conservative method to test awareness, forced choice recognition between the true prime and similar distractors.

In Experiments 1 and 2, the effect of subliminal priming on insight problem solving was examined using a short movie that included a hint that participants were not aware they had seen. In Experiment 3, a new method was used to address concerns about the methodology of Experiments 1 and 2.

\section{Experiment 1}

In the first experiment, we examined whether subliminal priming can affect human insight problem solving using the radiation problem. Half of participants were given an unrecognized hint during the attempt to solve the problem.

\section{Method}

\section{Participants and design}

A total of 206 undergraduate students ( 89 female and 117 male; age: $19-24$ years, $M=20.1, S D=1.1$ ) from Ritsumeikan University and Ryukoku University were tested. They were randomly assigned to either a hint or a nohint condition.

\section{Materials and apparatus}

The hint stimulus was presented as part of a 56-s movie, composed from one hint image (exposed $33 \mathrm{~ms} \times 60$ times), two mask images, three filler images, and one fixation image (Fig. 1; see the online materials for more detail). The movie

\footnotetext{
${ }^{1}$ Bowden (1997) revealed the effect of subliminal priming on anagrams. Anagrams, however, are not insight problems according to Weisberg's (1995) taxonomy, because solving an anagram does not require changes in the problem representation.
}

was projected on a screen at the front of the room by a liquid crystal display projector. The radiation problem was then presented in a booklet along with diagrams (available online). In the recognition task, participants were presented four figures (see Fig. 2), and their task was to choose the one that they thought had been shown with a confidence rating - "sure," "half-sure," and "guess".

\section{Procedure}

The experiment was administered in Japanese to groups in two different classrooms. Two minutes after participants started to solve the problem, they were asked to engage in an "irrelevant" task for $1 \mathrm{~min}$. While participants in the hint condition watched the hint movie (described as "an irrelevant short movie") on the screen, participants in the no-hint condition tackled the dummy calculation task (e.g., $23+18=$ ) to try to solve as many problems as possible while not looking at the screen. They were given a total of 9 min to complete the problem, including the initial 2-min trial period, the 1-min exposure or calculation period, and the second 6-min trial period. Finally, they were asked whether they have seen the radiation problem before and chose an answer among the options "yes," "no," and "I am not sure, but I might have seen it before."

\section{Results and discussion}

Of the 206 participants, 10 reported that they had seen the radiation problem before, 9 in the hint condition correctly identified the hint image with certainty, and 2 in the no-hint condition reported that they mistakenly had a glance at the hint movie. Data from all these participants were excluded from analysis. Of the remaining 185 participants, $56 \%$ $(49 / 88)$ solved the problem in the hint condition, whereas only $37 \%(36 / 97)$ succeeded in the no-hint condition, $\chi^{2}(1$, $N=185)=6.41, p=.01, \phi=.19$.

In the recognition task, $55 \%(48 / 88)$ selected the correct hint image ( 2 out of 88 did not answer) significantly more than chance (i.e., $25 \%$ ), $p<10^{-8}$, but the majority $(69 \%=33 / 48)$ of them reported that it was a "guess," while the others reported "half-sure." This means either that some of participants saw the hint with some degree of awareness or that they were biased to select the hint image. We did verify in pilot experiments that most people failed to recognize the hint. But if they saw it, correct responders in the recognition task should show a higher solution rate than incorrect responders. They did not. Solution rates for the two subgroups of the hint group were very close, $58 \%(28 / 48)$ versus $55 \%(21 / 38), \chi^{2}(1, N=86)=$ $0.082, p=.78, \phi=.03$. This suggests that correct identifiers did not have the hint image available to awareness 
Fig. 1 Schematic description of a sequence of frames including implicit hint stimulus presented in Experiments 1 (I) and 2 (II). These sequences (18 s long each) are looped three times with a 1-s blank between each loop and formed a total of a 56-s long movie that included 60 hint cuts

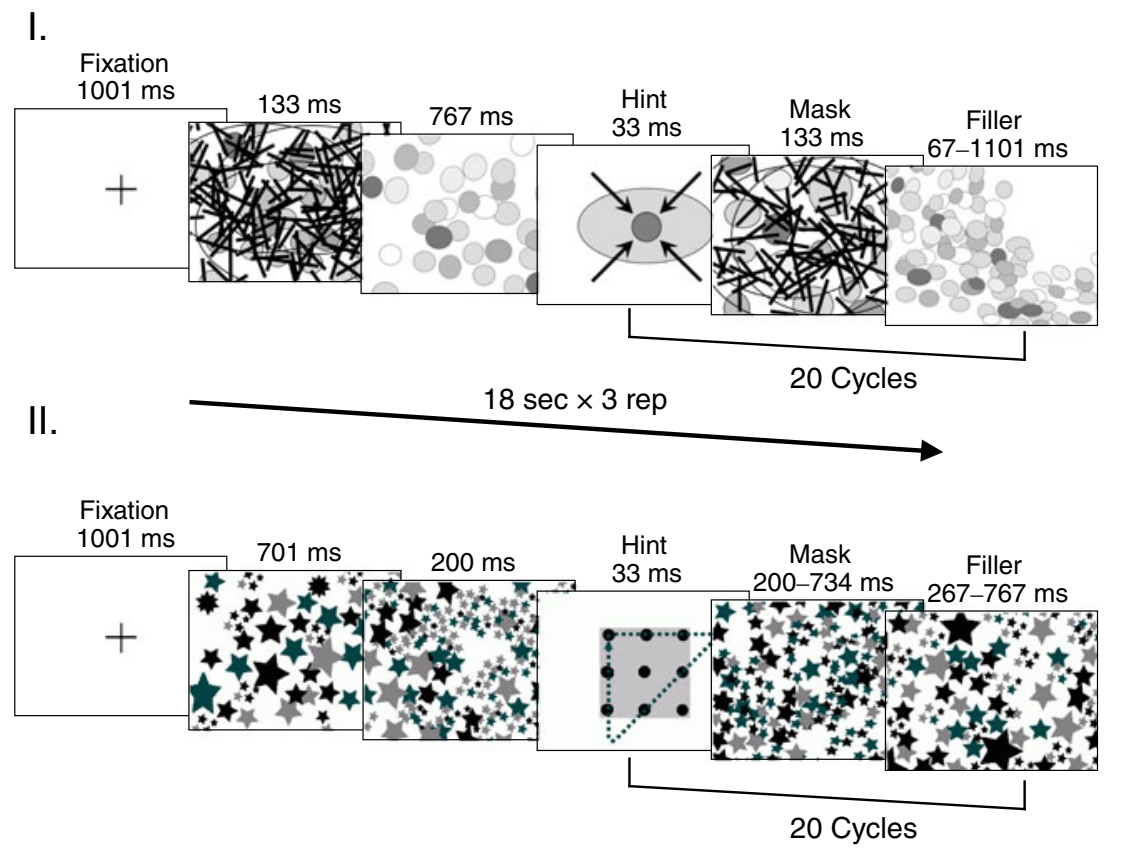

but, instead, were biased to choose it for some other reason. Perhaps it simply looked more plausible than the other images. ${ }^{2}$ In the next experiment, designed to examine the facilitation effect in a different problem, we examine this issue directly.

\section{Experiment 2}

In Experiment 1, an unrecognized hint raised the solution rate of the radiation problem by $50 \%$, suggesting that subliminal priming helped to solve an insight problem. In this experiment, we tried to replicate the results using a different problem that was less text based and more purely spatial, the nine-dot problem.

Method

\section{Participants and design}

A total of 133 undergraduate students from Brown University participated in the experiment. They were randomly assigned to either a hint or a no-hint condition. Of these, 4 did not follow the instructions correctly, and their data were excluded. The remaining 129 participants consisted of 59 female, 65 male, and 5 unknown (age range: $17-53$ years, $M=20.1, S D=3.2$ ).

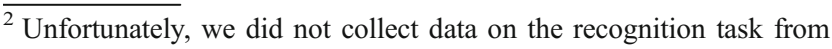
the no-hint group. Such data would have revealed whether there are biases in favor of one of the response options.
}

\section{Materials and apparatus}

A hint movie for the nine-dot problem and an answer booklet were used as in Experiment 1 (Fig. 1; see the online materials for details). The nine-dot problem was followed by a recognition inquiry (only for the hint condition), where participants were asked whether they saw the hint and they chose an answer from "yes," "no," and "I thought I saw something, but I didn't recognize it clearly." In the recognition task, they were given a forced choice among four figures - the true hint image and the identical image rotated by $90^{\circ}, 180^{\circ}$, and $270^{\circ}$.

\section{Procedure}

The experiment was administered to two groups in different classrooms, and the procedure was similar to Experiment 1. One minute after the experiment started, participants were shown the hint movie or given the dummy calculation task. They were given a total of $5 \mathrm{~min}$ to solve the problem.

\section{Results}

Of the 129 participants, 55 reported that they had seen the ninedot problem before, and 3 finished the task successfully within 1 min (i.e., before the exposure period). All their data were excluded from further analysis, unless otherwise stated. Of the remaining 71 participants, 2 out of the 28 participants tested in the hint condition reported having recognized the hint, but they all failed to correctly identify the true hint image. Solution rates in the hint and no-hint conditions were $29 \%(8 / 28)$ and $9 \%$ (4/43), respectively, $p=.05$ (Fisher's exact test), $\phi=.25$ (see Fig. 3). This result can be compared with an effect of a similar 
Fig. 2 Four alternatives prepared for the recognition task in Experiment 1. Participants forcedly chose one that they thought had been shown
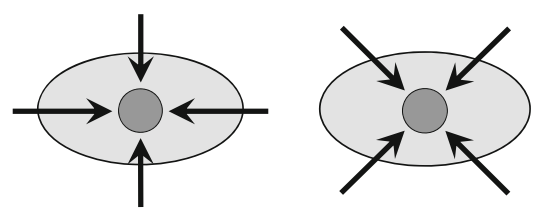

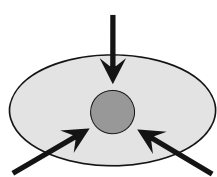

supraliminal hint reported by Chronicle, Ormerod, and MacGregor (2001), who used a shading pattern overlaid on the array of nine dots as a hint without mentioning its relevance. Their solution rate was $16 \%(5 / 31)$, a similar effect size, $\phi=$ $.29 .^{3}$

If the hint facilitates solving the problem, solutions should be similar to the one suggested by the hint. Figure 3 also shows the proportion of solutions that had the same structure as the hint, as opposed to one of the other three solutions (see Fig. 4). Of the successful solvers in the hint condition, $88 \%(7 / 8)$ matched the hint, whereas only $25 \%$ (1/4) of successful solvers did so in the no-hint condition, exactly what would be expected by chance. The difference between the two conditions was marginally significant, $p=.07$ (Fisher's exact test), although the effect size was large $\phi=.63$.

Results regarding participants' awareness were dissociated from the effect of the hint. Only 5 out of 57 participants (including the 27 who knew the problem and the 2 who finished within $1 \mathrm{~min}$ ) in the hint condition reported that they had recognized the hint, but 3 of the 5 failed to correctly identify the target in the recognition task. The distribution of their choices in the recognition task also indicated that they did not explicitly recognize the hint. The frequency of choosing each solution (Fig. 4) was 17, 13, 14, and 9, respectively (4 chose nothing), no different than a uniform distribution, $\chi^{2}(3, N=53)$ $=2.47, p=.48$. Recognition confidence also failed to predict the ability to solve the problem. The proportions correct were $40 \%(2 / 5), 22 \%(2 / 9)$, and $30 \%(13 / 43)$ for participants who reported "yes," "unsure," and "no" to the recognition inquiry, respectively, $p=.79$ (Fisher's exact test), Cramer's $V=.09$ (a very small effect size).

\section{Discussion}

The hint tripled the solution rate. Together with the results of Experiment 1, the results in the hint condition (i.e., a high solution rate, a high likelihood of solving the problem in a way consistent with the hint, and a low recognition rate) suggest an effect of subliminal stimulation. The method of Experiments 1 and 2 may raise some concerns, however. First, the effects might be caused by the mask or filler image instead of the hint. Second, insight might have been hampered by the calculation

\footnotetext{
${ }^{3}$ Since they actually did not include a control condition, we used a solution rate of the control group, $0 \%(0 / 27)$, reported in MacGregor, Ormerod, and Chronicle (2001).
}

task in the control conditions. These interpretations actually are not consistent with the finding that solutions matched the hint in the hint condition. Additionally, the latter interpretation is rendered suspect by the higher solution rates in the no-hint conditions (i.e., $37 \%$ and $9 \%$ ), as compared with the typical solution rates in the literature ( $9 \%$ and $4 \%$, respectively). ${ }^{4}$ However, we do not have direct evidence that the explicit images and the calculation task were irrelevant to the facilitation effect. Third, it might be regarded as a problem that all the participants did not receive exactly the same hint stimulus. That is, because the experiment was administered to participants as a group, the distance and angle to the hint varied with their seating positions. All of these concerns were addressed in the next experiment.

\section{Experiment 3}

In this experiment, the subliminal priming effect was examined by a more strictly controlled method than in the previous experiments. In order to generalize the results, we used a different insight problem than for previous experiments, the 10-coin problem. The problem is to turn a triangle composed of 10 coins upside down by moving no more than 3 coins (see Fig. 5).

\section{Method}

\section{Participants and design}

A total of 80 adults (39 female and 41 male; age: $18-25$ years including 1 unknown, $M=21.2, S D=1.6$ ) were tested. They were randomly assigned to either a hint or a no-hint condition. ${ }^{5}$

\footnotetext{
${ }^{4}$ The radiation problem is based on data from a total of 250 participants from 10 control conditions reported in Gick and Holyoak (1980), Spencer and Weisberg (1986), Holyoak and Koh (1987), and Thomas and Lleras $(2007,2009)$. The nine-dot problem is based on data from a total of 284 participants from seven control groups (or eliminated data) reported in Burnham and Davis (1969), Weisberg and Alba (1981), MacGregor et al. (2001), and Kershaw and Ohlsson (2004).

${ }^{5}$ Participants actually were assigned to one of four conditions: 2 (hint vs. no hint) $\times 2$ (instruction vs. no instruction). In the instruction conditions, they were encouraged to generate novel ideas. A directive like "Think unconventionally" was displayed on the center of the screen for $1 \mathrm{~s}$ immediately after each prime (Fig. 5). No message was displayed in the no-instruction condition. There was no main effect of the instruction, $\chi^{2}(1)=0.15, p=.71$, nor an interaction with the hint, $\chi^{2}(1)=0.15, p=.71$, by a two-way ANOVA based on a chi-square distribution. We therefore refrain from further discussion of this variable.
} 


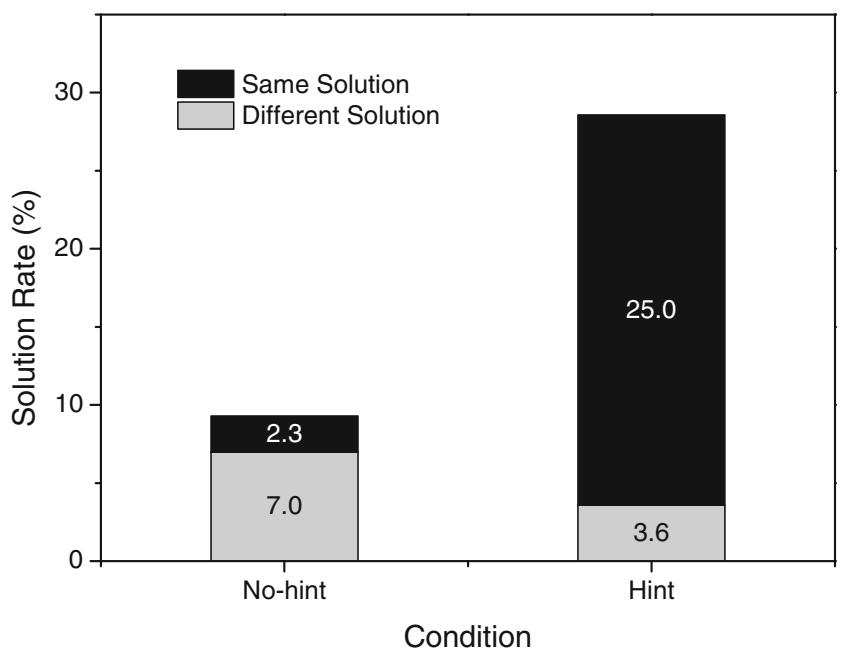

Fig. 3 Percentage of participants who solved the nine-dot problem in Experiment 2

\section{Materials and apparatus}

Participants solved the 10-coin problem on a 10.1-in. tablet computer (NEC PC-LT550FS) using a touch pen. All the operations, including receiving the hint and answering postquestions, were carried out on the device.

\section{Procedure}

The experiment was administered in Japanese individually. Participants were able to move any coins on the screen or to return to the initial state whenever they desired. In the hint condition, the hint was periodically displayed on the screen as the problem was solved (Fig. 5). In the no-hint condition, the hint was replaced by a blank screen. Before they started, participants read general instructions on the screen. They were notified in advance that they would see irregular polygons (i.e., a pattern mask) every $10 \mathrm{~s}$ and were falsely instructed that the experiment aimed to examine the effect of "irrelevant" visual stimuli during problem solving. The task ended when they successfully solved the problem. After they solved the problem or $4 \mathrm{~min}$ had passed, they were given several questions identical to those in Experiment 2 (see the online materials).

\section{Results and discussion}

Of the 80 participants, 3 reported that they had seen the 10 coin problem before, and 1 in the hint condition reported having recognized the hint. All their data were excluded from further analysis.

The solution rate was $26 \%(10 / 38)$ in the hint condition, but only $5 \%(2 / 38)$ in the no-hint condition, $\chi^{2}(1, N=76)=$ $6.33, p=.012, \phi=.29$. Figure 6 shows the cumulative distribution of successful solvers as time elapsed in each group. A log-rank test for equality of rise curves (i.e., survivor functions) showed a significant difference of solution times between the two groups, $\chi^{2}(1, N=76)=6.3$, $p=.01, \phi=.29$. In the recognition task (25\% chance level), only $26 \%(10 / 38)$ selected the correct answer, $p=.85$ (binomial test).

In sum, the effect of Experiments 1 and 2 was replicated. The hint quintupled the solution rate and shortened the solution time. The results suggest that the main cause of facilitation in the previous experiments was not explicit images given as masks or inexperience with the calculation task but the hint itself.

\section{General discussion}

In three experiments, we observed facilitation from subliminal priming on insight problem solving. Hints increased solution rates in all three experiments, despite participants being both subjectively unaware of primes and also unable to confidently discriminate the target from distractors.

The results shed light on how impasses are resolved. The subliminal priming effect is more consistent with theories based on activation in memory, including the redistribution theory of insight (Ohlsson, 2011), than with those based on awareness. Exposure to a hint can activate insightful ideas without awareness, increasing the probability of producing a corresponding strategy and of deactivating inappropriate ones to escape from the impasse.

One condition likely critical to the effect of subliminal priming is preparedness. Moss, Kotovsky, and Cagan (2007) obtained evidence that open goals set in a task (i.e.,

Fig. 4 Four solutions of the nine-dot problem. Only solution 1 matches to the implicit hint

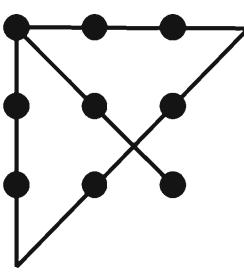

Solution 1

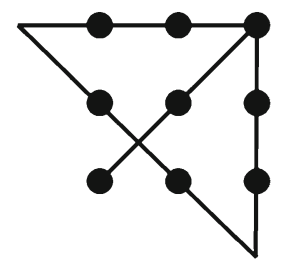

Solution 2

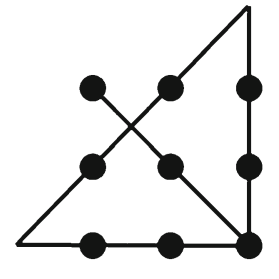

Solution 3

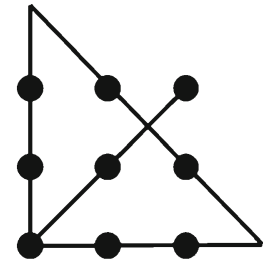

Solution 4 
Fig. 5 Schematic depiction of the temporal task structure in Experiment 3. Actual duration time of each image was measured by counting the number of frames (600 fps) captured by a high-speed camera (Casio EX-F1). The means and standard deviations of values obtained by 10 measurements are shown $(M \pm$ $S D)$. In the no-hint condition, each hint image was replaced by a blank screen $(23 \pm 6 \mathrm{~ms}$ each)

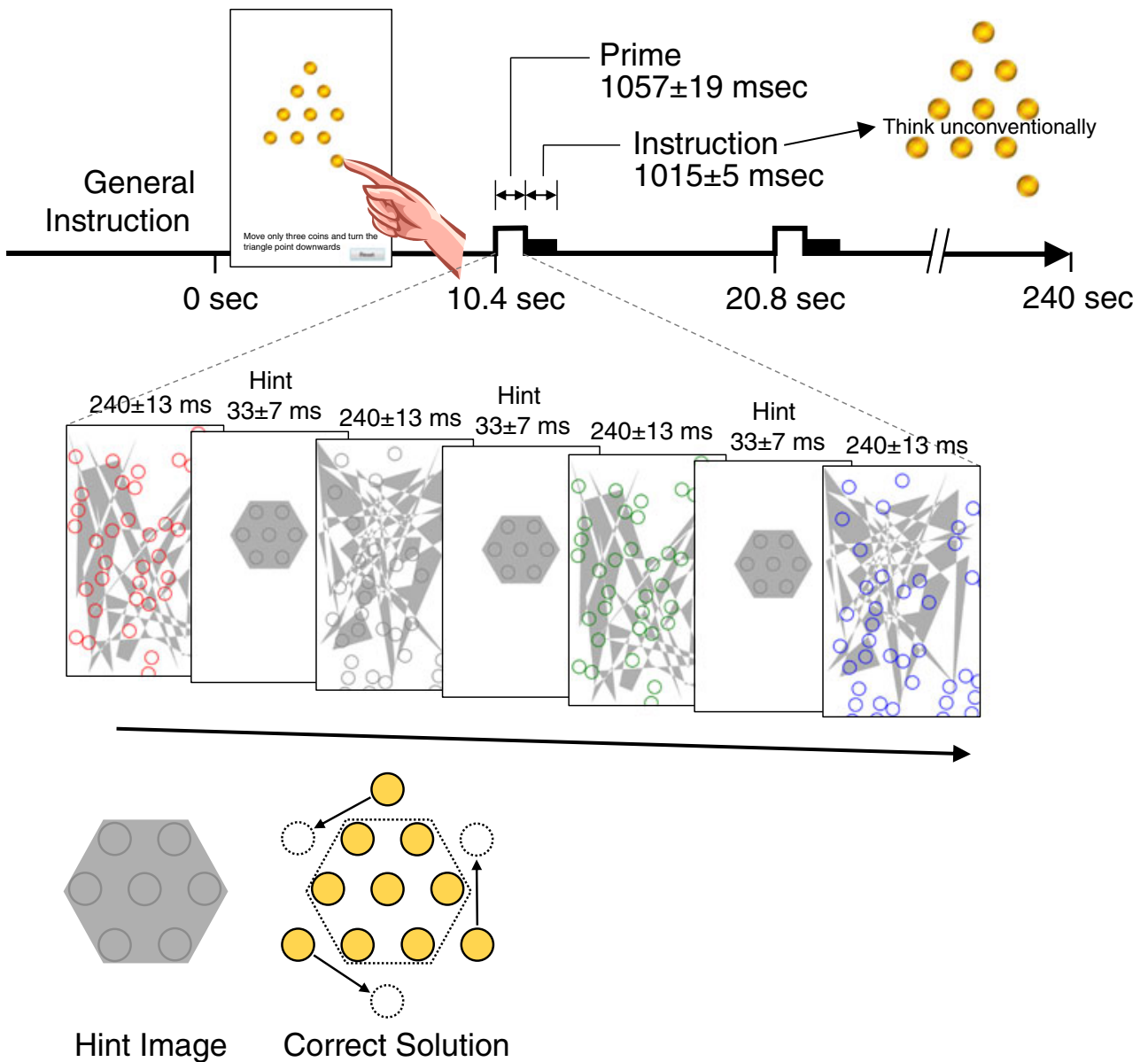

unsolved problems) promoted acquisition of hints implicitly presented in another task. The opportunistic assimilation hypothesis (Seifert et al., 1995) supposes that reaching impasse sets up "failure indices" in memory and relevant information later introduced in the environment may lead to retrieval of these indices, which may result in an insight.

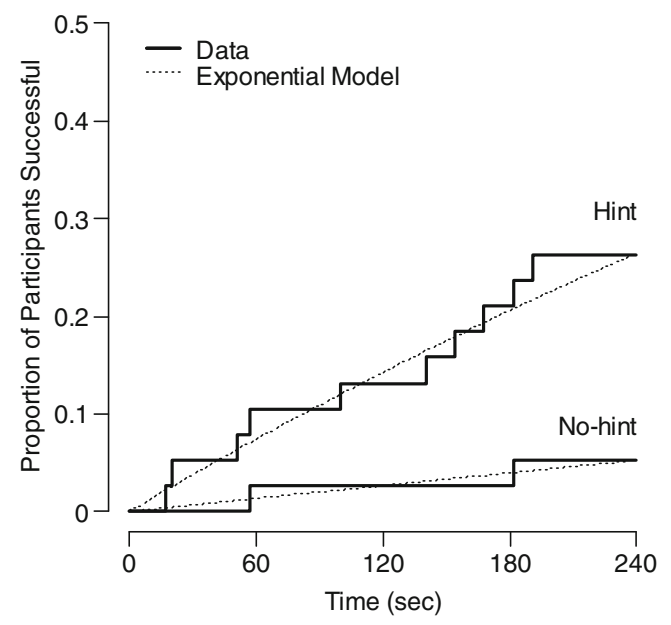

Fig. 6 Proportion of participants who successfully solved the 10-coin problem, with time in each condition
So the procedure we used in Experiments 1 and 2 (i.e., presenting the hint a few minutes after participants started tackling the problem) might have made participants receptive to priming. Waiting a few minutes allowed them to set their goals. Indeed, the effectiveness of incubation periods in creative thinking could involve such goal setting.

Psychologists have argued about whether insights are initially unconscious or not. Some theorists have claimed that "insights are not always conscious from the start" (Siegler, 2000, p. 82) or that first, "unconscious thought 'boosts' the associative search for creative solutions" and then "solutions are transferred to consciousness" (Zhong, Dijksterhuis, \& Galinsky, 2008, p. 916). Such claims are consistent with our present data, but such processes may require enabling conditions and the absence of disablers. Conscious verbal processes can interfere with unconscious processes in insight problem solving (Schooler, Ohlsson, \& Brooks, 1993). Moreover, although conscious control alone is known to facilitate creativity (e.g., Nickerson, 1999), intentional activities can hamper unconscious processes. Mindful students in a classroom were insensitive to subliminal priming (Radel et al., 2009), and similarly, conscious attention eliminated priming effects on social perception (Dijksterhuis \& Van Knippenberg, 2000). How priming 
effects or unconscious processes interact with more intentional and controlled activities is an important open issue. Unconscious processing in insight problem solving cannot be studied by methods like verbal self-report that require conscious processing. Thus, we must rely on experimental methods that tap implicit processes, like the subliminal priming technique.

Acknowledgements We thank Phil Fernbach, and Hiroaki Suzuki for their helpful comments on this study. We are also grateful to Yuriko Shibata for her help in developing experimental materials in a pilot study.

This research was supported by Grant-in-Aid for Scientific Research 22500247 from Japan Society for the Promotion of Science and a research grant from the NeuroCreative Lab (NPO) to M.H.

\section{References}

Bargh, J. A., \& Pietromonaco, P. (1982). Automatic information processing and social perception: The influence of trait information presented outside of conscious awareness on impression formation. Journal of Personality and Social Psychology, 43, 437-449. doi:10.1037/0022-3514.43.3.437

Bowden, E. M. (1997). The effect of reportable and unreportable hints on anagram solution and the aha! experience. Consciousness and Cognition, 6, 545-573. doi:10.1006/ccog.1997.0325

Burnham, C. A., \& Davis, K. G. (1969). The nine-dot problem: Beyond perceptual organisation. Psyochonomic Science, 17, 321-323.

Chronicle, E. P., MacGregor, J. N., \& Ormerod, T. C. (2004). What makes an insight problem? The role of heuristics, goal conception, and solution recoding in knowledge-lean problems. Journal of Experimental Psychology: Learning, Memory, and Cognition, 30, 14-27. doi:10.1037/0278-7393.30.1.14

Chronicle, E. P., Ormerod, T. C., \& MacGregor, J. N. (2001). When insight just won't come: The failure of visual cues in the nine-dot problem. The Quarterly Journal of Experimental Psychology, 54A, 903-919. doi:10.1080/02724980042000471

Dijksterhuis, A., \& Van Knippenberg, A. (2000). Behavioral indecision: Effects of self-focus on automatic behavior. Social Cognition, 18, 55-74. doi:10.1521/soco.2000.18.1.55

Duncker, K. (1945). On problem-solving. Psychological Monographs, 58(5), i-113. doi:10.1037/h0093599

Gick, M. L., \& Holyoak, K. J. (1980). Analogical problem solving. Cognitive Psychology, 12, 306-355.

Holender, D. (1986). Semantic activation without conscious identification in dichotic listening, parafoveal vision, and visual masking: A survey and appraisal. Behavioral and Brain Sciences, 9, 1-23. doi:10.1017/S0140525X00021269

Holyoak, K., \& Koh, K. (1987). Surface and structural similarity in analogical transfer. Memory \& Cognition, 15, 332-340. doi:10.3758/bf03197035

Kaplan, C. A., \& Simon, H. A. (1990). In search of insight. Cognitive Psychology, 22, 374-419. doi:10.1016/0010-0285(90)90008-R

Karremans, J. C., Stroebe, W., \& Claus, J. (2006). Beyond Vicary's fantasies: The impact of subliminal priming and brand choice. Journal of Experimental Social Psychology, 42, 792-798. doi:10.1016/j.jesp.2005.12.002

Kershaw, T. C., \& Ohlsson, S. (2004). Multiple causes of difficulty in insight: The case of the nine-dot problem. Journal of Experimental Psychology: Learning, Memory, and Cognition, 30, 3-13. doi:10.1037/0278-7393.30.1.3
Kunst-Wilson, W. R., \& Zajonc, R. B. (1980). Affective discrimination of stimuli that cannot be recognized. Science, 207, 557-558. doi:10.1126/science. 7352271

Légal, J.-B., Chappé, J., Coiffard, V., \& Villard-Forest, A. (2012). Don't you know that you want to trust me? Subliminal goal priming and persuasion. Journal of Experimental Social Psychology, 48, 358-360. doi:10.1016/ j.jesp.2011.06.006

Lockhart, R. S., Lamon, M., \& Gick, M. L. (1988). Conceptual transfer in simple insight problems. Memory \& Cognition, 16, 36-44. doi:10.3758/BF03197743

MacGregor, J. N., Ormerod, T. C., \& Chronicle, E. P. (2001). Information processing and insight: A process model of performance on the nine-dot and related problems. Journal of Experimental Psychology: Learning, Memory, and Cognition, 27, 176-201. doi:10.1037//0278-7393.27.1.176

Maier, N. R. F. (1931). Reasoning in humans: II. The solution of a problem and its appearance in consciousness. Journal of Comparative Psychology, 12, 181-194. doi:10.1037/ h0071361

Moss, J., Kotovsky, K., \& Cagan, J. (2007). The influence of open goals on the acquisition of problem-relevant information. Journal of Experimental Psychology: Learning, Memory, and Cognition, 33, 876-891. doi:10.1037/02787393.33.5.876

Nickerson, R. S. (1999). Enhancing creativity. In R. J. Sternberg (Ed.), Handbook of creativity (pp. 392-430). Cambridge, UK: Cambridge University Press.

Nishimura, Y., \& Suzuki, H. (2006). Dousatsu mondai kaiketsu-no seiyaku kanwa-ni okeru senzaiteki jouhou syori [Implicit information processing of constraint relaxation in insight problemsolving]. Cognitive Studies, 13, 136-138.

Ohlsson, S. (2011). Deep learning: How the mind overrides experience. New York, NY, US: Cambridge University Press.

Radel, R., Sarrazin, P., Legrain, P., \& Gobancé, L. (2009). Subliminal priming of motivational orientation in educational settings: Effect on academic performance moderated by mindfulness. Journal of Research in Personality, 43, 695-698. doi:10.1016/ j.jrp.2009.02.011

Schooler, J. W., Ohlsson, S., \& Brooks, K. (1993). Thoughts beyond words: When language overshadows insight. Journal of Experimental Psychology: General, 122, 166-183. doi:10.1037/ 0096-3445.122.2.166

Schunn, C. D., \& Dunbar, K. (1996). Priming, analogy, and awareness in complex reasoning. Memory \& Cognition, 24, 271-284. doi:10.3758/BF03213292

Seifert, C. M., Meyer, D. E., Davidson, N. S., Patalano, A. L., \& Yaniv, I. (1995). Demystification of cognitive insight: Opportunistic assimilation and the prepared-mind hypothesis. In R. J. Sternberg \& J. E. Davidson (Eds.), The nature of insight (pp. 65-124). Cambridge, MA: MIT Press.

Siegler, R. S. (2000). Unconscious insights. Current Directions in Psychological Science, 9, 79-83. doi:10.1111/14678721.00065

Snodgrass, M., Bernat, E., \& Shevrin, H. (2004). Unconscious perception: A model-based approach to method and evidence. Perception \& Psychophysics, 66, 846-867. doi:10.3758/ BF03194978

Spencer, R. M., \& Weisberg, R. W. (1986). Context-dependent effects on analogical transfer. Memory \& Cognition, 14, 442-449. doi:10.3758/bf03197019

Thomas, L. E., \& Lleras, A. (2007). Moving eyes and moving thought: On the spatial compatibility between eye movements and cognition. Psychonomic Bulletin \& Review, 14, 663-668. doi:10.3758/ BF03196818 
Thomas, L. E., \& Lleras, A. (2009). Covert shifts of attention function as an implicit aid to insight. Cognition, 111, 168-174. doi:10.1016/j.cognition.2009.01.005

Weisberg, R. W. (1995). Prolegomena to theories of insight in problem solving: A taxonomy of problems. In R. J. Sternberg \& J. E. Davidson (Eds.), The nature of insight (pp. 157-196). Cambridge, MA: MIT Press.
Weisberg, R. W., \& Alba, J. W. (1981). Gestalt theory, insight, and past experience: Reply to Dominowski. Journal of Experimental Psychology: General, 110, 193-198. doi:10.1037/0096-3445. 110.2.199

Zhong, C.-B., Dijksterhuis, A., \& Galinsky, A. D. (2008). The merits of unconscious thought in creativity. Psychological Science, 19, 912-918. doi:10.1111/j.1467-9280.2008.02176.x 